\title{
EXTENSION GROUPS FOR DG MODULES
}

\author{
SAEED NASSEH AND SEAN SATHER-WAGSTAFF
}

\begin{abstract}
Let $M$ and $N$ be differential graded (DG) modules over a positively graded commutative DG algebra $A$. We show that the Ext-groups $\operatorname{Ext}_{A}^{i}(M, N)$ defined in terms of semi-projective resolutions are not in general isomorphic to the Yoneda Ext-groups $\operatorname{YExt}_{A}^{i}(M, N)$ given in terms of equivalence classes of extensions. On the other hand, we show that these groups are isomorphic when the first DG module is semi-projective.
\end{abstract}

\section{INTRODUCTION}

Convention. In this paper, $R$ is a commutative ring with identity.

Given two $R$-modules $M$ and $N$, a classical result originating with work of Baer 4 states that $\operatorname{Ext}_{R}^{1}(M, N)$, defined via projective/injective resolutions, is isomorphic to the abelian group $\operatorname{YExt}_{R}^{1}(M, N)$ of equivalence classes of exact sequences of the form $0 \rightarrow N \rightarrow X \rightarrow M \rightarrow 0$. The purpose of this note is to discuss possible extensions of this result to the abelian category of differential graded (DG) modules over a positively graded commutative DG algebra $A$. See Section 2 for background information on this category.

Specifically, we show that Baer's isomorphism fails in general in this context: Examples 3.1 and 3.2 exhibit DG $A$-modules $M, N$ with $\operatorname{Ext}_{A}^{1}(M, N) \supsetneqq \operatorname{YExt}_{A}^{1}(M, N)$. (See2.4 and 2.6 below for definitions.) On the other hand, the following result shows that a reasonable hypothesis on the first module does yield such an isomorphism.

Theorem A. Let $A$ be a $D G R$-algebra, and let $N, Q$ be $D G A$-modules such that $Q$ is semi-projective. Then there is an isomorphism $\operatorname{YExt}_{A}^{i}(Q, N) \cong \operatorname{Ext}_{A}^{i}(Q, N)$ of abelian groups for all $i \geqslant 1$.

This is the main result of Section 3, see Proof 3.8. In the subsequent Section 4 we discuss some properties of YExt with respect to truncations.

It is worth noting here that we apply results from this paper in our answer to a question of Vasconcelos in 12. Specifically, in that paper, we investigate DG $A$ modules $C$ with $\operatorname{Ext}_{A}^{1}(C, C)=0$ using geometric techniques. These techniques yield an isomorphism between $\operatorname{YExt}_{A}^{1}(C, C)$ and a certain quotient of tangent spaces; it is then important for us to know when the vanishing of $\operatorname{Ext}_{A}^{1}(C, C)$ implies the vanishing of related YExt ${ }^{1}$-modules; see Proposition 4.4 below.

2010 Mathematics Subject Classification. 13D02, 13D07, 13D09.

Key words and phrases. Differential graded algebras, differential graded modules, Yoneda Ext. Sather-Wagstaff was supported in part by North Dakota EPSCoR, National Science Foundation Grant EPS-0814442, and NSA grant H98230-13-1-0215. 


\section{DG Modules}

We assume that the reader is familiar with the category of $R$-complexes and the derived category $\mathcal{D}(R)$. Standard references for these topics are [6, 7, 9, 10, 13, 14]. For clarity, we include some definitions and notation.

Definition 2.1. In this paper, complexes of $R$-modules (" $R$-complexes" for short) are indexed homologically: $M=\cdots \stackrel{\partial_{n+2}^{M}}{\longrightarrow} M_{n+1} \stackrel{\partial_{n+1}^{M}}{\longrightarrow} M_{n} \stackrel{\partial_{n}^{M}}{\longrightarrow} M_{n-1} \stackrel{\partial_{n-1}^{M}}{\longrightarrow} \cdots$. The degree of an element $m \in M$ is denoted $|m|$. The infimum and supremum of $M$ are the infimum and supremum, respectively, of the set $\left\{n \in \mathbb{Z} \mid \mathrm{H}_{n}(M) \neq 0\right\}$. The tensor product of two $R$-complexes $M, N$ is denoted $M \otimes_{R} N$, and the Hom complex is denoted $\operatorname{Hom}_{R}(M, N)$. A chain map $M \rightarrow N$ is a cycle in $\operatorname{Hom}_{R}(M, N)_{0}$.

Next we discuss DG algebras and DG modules, which are treated in, e.g., 1, 2, 3. 5, 8, 11]. We follow the notation and terminology from [2, 5]; given the slight differences in the literature, though, we include a summary next.

Definition 2.2. A positively graded commutative differential graded $R$-algebra ( $D G$ $R$-algebra for short) is an $R$-complex $A$ equipped with a chain map $\mu^{A}: A \otimes_{R} A \rightarrow A$ with $a b:=\mu^{A}(a \otimes b)$ that is associative, unital, and graded commutative such that $A_{i}=0$ for $i<0$. The map $\mu^{A}$ is the product on $A$. Given a DG $R$-algebra $A$, the underlying algebra is the graded commutative $R$-algebra $A^{\natural}=\oplus_{i \geqslant 0} A_{i}$.

A differential graded module over a DG $R$-algebra $A$ ( $D G A$-module for short) is an $R$-complex $M$ with a chain map $\mu^{M}: A \otimes_{R} M \rightarrow M$ such that the rule $a m:=\mu^{M}(a \otimes m)$ is associative and unital. The map $\mu^{M}$ is the scalar multiplication on $M$. The underlying $A^{\natural}$-module associated to $M$ is the $A^{\natural}$-module $M^{\natural}=\oplus_{i \in \mathbb{Z}} M_{i}$.

The DG $A$-module $\operatorname{Hom}_{A}(M, N)$ is the subcomplex of $\operatorname{Hom}_{R}(M, N)$ of the $A$ linear homomorphisms. A morphism $M \rightarrow N$ of DG $A$-modules is a cycle in $\operatorname{Hom}_{A}(M, N)_{0}$. Projective objects in the category of DG $A$-modules are called categorically projective. Quasiisomorphisms of DG $A$-modules are identified by the symbol $\simeq$, also used for the "quasiisomorphic" equivalence relation.

Two important DG $R$-algebras to keep in mind are $R$ itself and, more generally, the Koszul complex over $R$ (on a finite sequence of elements of $R$ ) with the exterior product. A DG $R$-module is just an $R$-complex, and a morphism of DG $R$-modules is simply a chain map.

Remark 2.3. Let $A$ be a DG $R$-algebra. The category of DG $A$-modules is an abelian category with enough projectives.

Definition 2.4. Let $A$ be a DG $R$-algebra, and let $M, N$ be DG $A$-modules. For each $i \geqslant 0$ we have a well-defined Yoneda Ext group $\operatorname{YExt}_{A}^{i}(M, N)$, defined in terms of a resolution of $M$ by categorically projective DG $A$-modules:

$$
\cdots \rightarrow Q_{1} \rightarrow Q_{0} \rightarrow M \rightarrow 0 .
$$

A standard result shows that $\operatorname{YExt}_{A}^{1}(M, N)$ is isomorphic to the set of equivalence classes of exact sequences $0 \rightarrow N \rightarrow X \rightarrow M \rightarrow 0$ of DG $A$-modules under the Baer sum; see, e.g., [15, (3.4.6)] and the proof of Theorem 3.5 .

We now turn to the derived category $\mathcal{D}(A)$, and related notions.

Definition 2.5. Let $A$ be a DG $R$-algebra. A DG $A$-module $Q$ is graded-projective if $\operatorname{Hom}_{A}(Q,-)$ preserves surjective morphisms, that is, if $Q^{\natural}$ is a projective graded 
$R^{\natural}$-module. The DG module $Q$ is semi-projective if $\operatorname{Hom}_{A}(Q,-)$ respects surjective quasiisomorphisms, that is, if $Q$ is graded-projective and respects quasiisomorphisms. A semi-projective resolution of $M$ is a quasiisomorphism $L \stackrel{\simeq}{\rightarrow} M$ of DG $A$-modules such that $L$ is semi-projective.

Fact 2.6. Let $A$ be a DG $R$-algebra. Then every DG $A$-module has a semiprojective resolution.

Definition 2.7. Let $A$ be a DG $R$-algebra. The derived category $\mathcal{D}(A)$ is formed from the category of DG $A$-modules by formally inverting the quasiisomorphisms; see 11. Isomorphisms in $\mathcal{D}(A)$ are identified by the symbol $\simeq$.

The derived functor $\mathbf{R H o m}_{A}(M, N)$ is defined via a semi-projective resolution $P \stackrel{\simeq}{\rightarrow} M$, as $\mathbf{R H o m}_{A}(M, N) \simeq \operatorname{Hom}_{A}(P, N)$. For each $i \in \mathbb{Z}$, set $\operatorname{Ext}_{A}^{i}(M, N):=$ $\mathrm{H}_{-i}\left(\mathbf{R H o m}_{A}(M, N)\right)$.

\section{DG Ext vs. Yoneda Ext}

We begin this section with examples of DG $A$-modules $M$ and $N$ such that $\operatorname{Ext}_{A}^{1}(M, N) \nsucceq \operatorname{YExt}_{A}^{1}(M, N)$. These present two facets of the distinctness of Ext and YExt, as the first example has $M$ and $N$ both bounded, while the second one (from personal communication with Avramov) has $M$ graded-projective.

Example 3.1. Let $R=k \llbracket X \rrbracket$, and consider the following exact sequence of DG $R$-modules, i.e., exact sequence of $R$-complexes:

$$
0 \longrightarrow \underline{R} \longrightarrow \underline{R} \longrightarrow \underline{k} \longrightarrow 0
$$

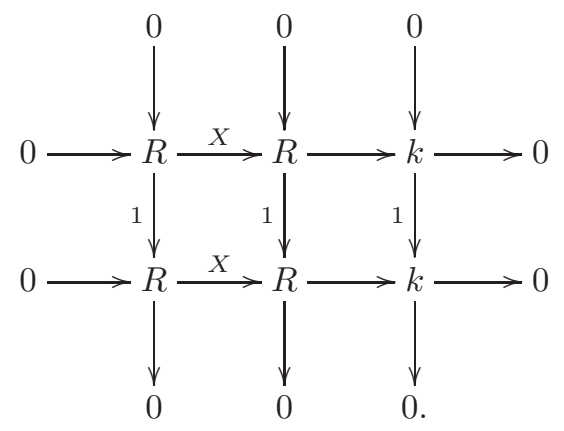

This sequence does not split over $R$ (it is not even degree-wise split) so it gives a non-trivial class in $\operatorname{YExt}_{R}^{1}(\underline{k}, \underline{R})$, and we conclude that $\operatorname{YExt}_{R}^{1}(\underline{k}, \underline{R}) \neq 0$. On the other hand, $\underline{k}$ is homologically trivial, so we have $\operatorname{Ext}_{R}^{1}(\underline{k}, \underline{R})=0$ since 0 is a semi-free resolution of $\underline{k}$.

Example 3.2. Let $R=k[X] /\left(X^{2}\right)$ and consider the following exact gradedprojective DG $R$-module $M=\cdots \stackrel{X}{\rightarrow} R \stackrel{X}{\rightarrow} R \stackrel{X}{\rightarrow} \cdots$. Since $M$ is exact, we have $\operatorname{Ext}_{R}^{i}(M, M)=0$ for all $i$. We claim, however, that $\operatorname{YExt}_{R}^{1}(M, M) \neq 0$. To see this, first note that $M$ is isomorphic to the suspension $\Sigma M$ and that $M$ is not contractible. Thus, the mapping cone sequence for the identity morphism $\operatorname{id}_{M}$ is isomorphic to one of the form $0 \rightarrow M \rightarrow X \rightarrow M \rightarrow 0$ and is not split. 
The definition of the isomorphism $\operatorname{YExt}_{A}^{i}(Q, N) \rightarrow \operatorname{Ext}_{A}^{i}(Q, N)$ for $i=1$ in Theorem $\mathrm{A}$ is contained in the following construction. The subsequent lemma and theorem show that $\Psi$ is a well-defined isomorphism.

Construction 3.3. Let $A$ be a DG $R$-algebra, and let $N, Q$ be DG $A$-modules such that $Q$ is graded-projective. Define $\Psi: \operatorname{YExt}_{A}^{1}(Q, N) \rightarrow \mathrm{H}_{-1}\left(\operatorname{Hom}_{A}(Q, N)\right)$ as follows. Note that if $Q$ is semi-projective, then $\operatorname{Ext}_{A}^{1}(Q, N) \cong \mathrm{H}_{-1}\left(\operatorname{Hom}_{A}(Q, N)\right)$, which fits with what we have in Theorem A

Let $\zeta \in \operatorname{YExt}_{A}^{1}(Q, N)$ be represented by the sequence

$$
0 \rightarrow N \stackrel{f}{\rightarrow} X \stackrel{g}{\rightarrow} Q \rightarrow 0
$$

Since $Q$ is graded-projective, this sequence is graded-split, that is there are elements $h \in \operatorname{Hom}_{A}(X, N)_{0}$ and $k \in \operatorname{Hom}_{A}(Q, X)_{0}$ with

$$
h f=\operatorname{id}_{N} \quad g k=\operatorname{id}_{Q} \quad h k=0 \quad f h+k g=\mathrm{id}_{X} .
$$

Thus, the sequence (3.3.1) is isomorphic to one of the form

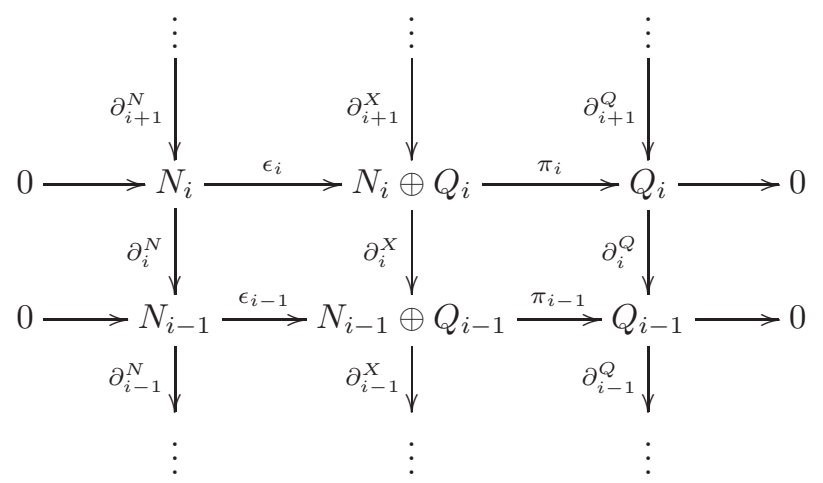

where $\epsilon_{j}$ is the natural inclusion and $\pi_{j}$ is the natural surjection for each $j$. Since this diagram comes from a graded-splitting of (3.3.1), the scalar multiplication on the middle column of $(3.3 .2)$ is the natural one $a\left[\begin{array}{c}p \\ q\end{array}\right]=\left[\begin{array}{l}a p \\ a q\end{array}\right]$. (We write elements of $N_{i} \oplus Q_{i}$ as column vectors.)

The fact that (3.3.2) commutes implies that $\partial_{i}^{X}$ has a specific form:

$$
\partial_{i}^{X}=\left[\begin{array}{cc}
\partial_{i}^{N} & \lambda_{i} \\
0 & \partial_{i}^{Q}
\end{array}\right]
$$

Here, we have $\lambda_{i}: Q_{i} \rightarrow N_{i-1}$, that is, $\lambda=\left\{\lambda_{i}\right\} \in \operatorname{Hom}_{R}(Q, N)_{-1}$. Since the horizontal maps in the sequence (3.3.2) are morphisms of DG $A$-modules, it follows that $\lambda$ is a cycle in $\operatorname{Hom}_{A}(Q, N)_{-1}$. Thus, $\lambda$ represents a homology class in $\mathrm{H}_{-1}\left(\operatorname{Hom}_{A}(Q, N)\right)$, and we define $\Psi: \operatorname{YExt}_{A}^{1}(Q, N) \rightarrow \mathrm{H}_{-1}\left(\operatorname{Hom}_{A}(Q, N)\right)$ by setting $\Psi(\zeta)$ equal to $[\lambda]$ the homology class of $\lambda$ in $\mathrm{H}_{-1}\left(\operatorname{Hom}_{A}(Q, N)\right)$.

Lemma 3.4. Let $A$ be a $D G R$-algebra, and let $N, Q$ be $D G$ A-modules such that $Q$ is graded-projective. Then the map $\Psi: \operatorname{YExt}_{A}^{1}(Q, N) \rightarrow \mathrm{H}_{-1}\left(\operatorname{Hom}_{A}(Q, N)\right)$ from Construction 3.3 is well-defined. 
Proof. Let $\zeta \in \operatorname{YExt}_{A}^{1}(Q, N)$ be represented by the sequence (3.3.2), and let $\zeta$ be represented by another exact sequence

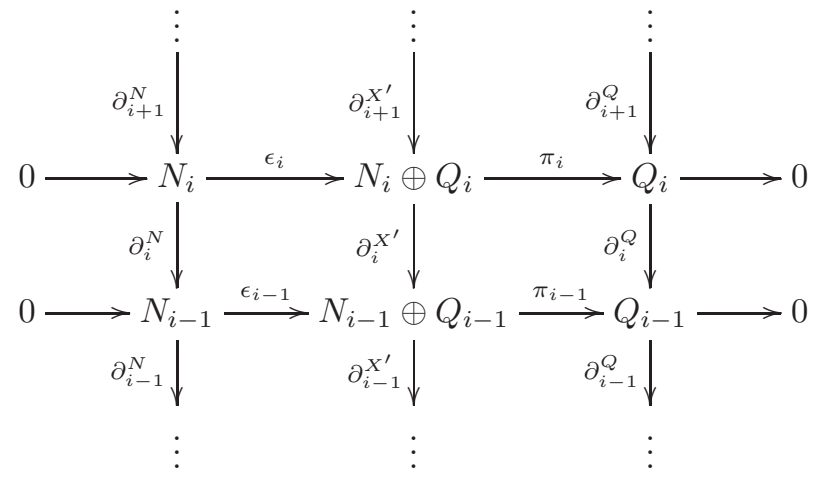

where

$$
\partial_{i}^{X^{\prime}}=\left[\begin{array}{cc}
\partial_{i}^{N} & \lambda_{i}^{\prime} \\
0 & \partial_{i}^{Q}
\end{array}\right]
$$

We need to show that $\lambda-\lambda^{\prime} \in \operatorname{Im}\left(\partial_{0}^{\operatorname{Hom}_{A}(Q, N)}\right)$. The sequences (3.3.2) and (3.4.1) are equivalent in $\operatorname{YExt}_{R}^{1}(Q, N)$, so for each $i$ there is a commutative diagram

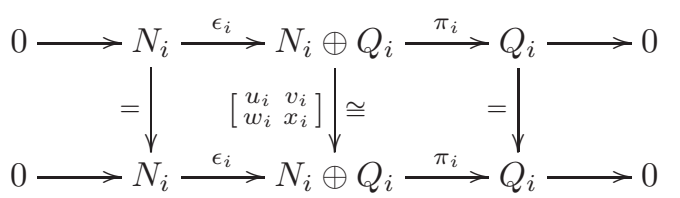

where the middle vertical arrow describes a DG $A$-module isomorphism, and such that the following diagram commutes for all $i$

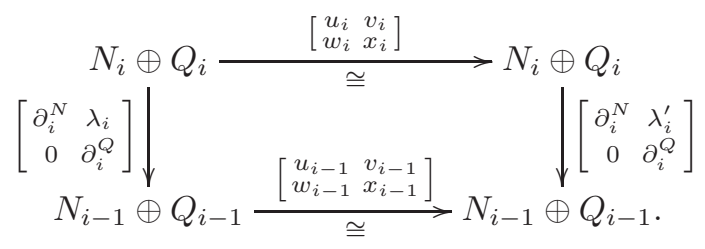

The fact that diagram (3.4.3) commutes implies that $u_{i}=\mathrm{id}_{N_{i}}, x_{i}=\mathrm{id}_{Q_{i}}$, and $w_{i}=0$. Also, the fact that the middle vertical arrow in diagram (3.4.3) describes a DG $A$-module morphism implies that the sequence $v_{i}: Q_{i} \rightarrow N_{i}$ respects scalar multiplication, i.e., we have $v \in \operatorname{Hom}_{A}(Q, N)_{0}$. The fact that diagram (3.4.4) commutes implies that $\lambda_{i}-\lambda_{i}^{\prime}=\partial_{i}^{N} v_{i}-v_{i-1} \partial_{i}^{Q}$. We conclude that $\lambda-\lambda^{\prime}=$ $\partial_{0}^{\mathrm{Hom}_{A}(Q, N)}(v) \in \operatorname{Im}\left(\partial_{0}^{\mathrm{Hom}_{A}(Q, N)}\right)$, so $\Psi$ is well-defined.

The next result contains the case $i=1$ of Theorem $\mathrm{A}$ from the introduction, because if $Q$ is semi-projective, then $\operatorname{Ext}_{A}^{1}(Q, N) \cong \mathrm{H}_{-1}\left(\operatorname{Hom}_{A}(Q, N)\right)$.

Theorem 3.5. Let $A$ be a $D G R$-algebra, and let $N, Q$ be $D G A$-modules such that $Q$ is graded-projective. Then the map $\Psi: \operatorname{YExt}_{A}^{1}(Q, N) \rightarrow \mathrm{H}_{-1}\left(\operatorname{Hom}_{A}(Q, N)\right)$ from Construction 3.3 is a group isomorphism.

Proof. We break the proof into three claims. 
Claim 1. $\Psi$ is additive. Let $\zeta, \zeta^{\prime} \in \operatorname{YExt}_{A}^{1}(Q, N)$ be represented by exact sequences $0 \rightarrow N \stackrel{\epsilon}{\rightarrow} X \stackrel{\pi}{\rightarrow} Q \rightarrow 0$ and $0 \rightarrow N \stackrel{\epsilon^{\prime}}{\rightarrow} X^{\prime} \stackrel{\pi^{\prime}}{\rightarrow} Q \rightarrow 0$ respectively, where $X_{i}=$ $N_{i} \oplus Q_{i}=X_{i}^{\prime}$ and the differentials $\partial^{X}$ and $\partial^{X^{\prime}}$ are described as in (3.3.3) and (3.4.2), respectively. We need to show that the Baer sum $\zeta+\zeta^{\prime}$ is represented by an exact sequence $0 \rightarrow N \stackrel{\widetilde{\epsilon}}{\rightarrow} \widetilde{X} \stackrel{\widetilde{\pi}}{\rightarrow} Q \rightarrow 0$, where $\widetilde{X}_{i}=N_{i} \oplus Q_{i}$ and $\partial_{i}^{\widetilde{X}}=\left[\begin{array}{cc}\partial_{i}^{N} \lambda_{i}+\lambda_{i}^{\prime} \\ 0 & \partial_{i}^{Q}\end{array}\right]$, with scalar multiplication $a\left[\begin{array}{l}p \\ q\end{array}\right]=\left[\begin{array}{l}a p \\ a q\end{array}\right]$. Note that it is straightforward to show that the sequence $\widetilde{X}$ defined in this way is a DG $A$-module, and the natural maps $N \stackrel{\widetilde{\epsilon}}{\rightarrow} \widetilde{X} \stackrel{\widetilde{\pi}}{\rightarrow} Q$ are $A$-linear, using the analogous properties for $X$ and $X^{\prime}$.

We construct the Baer sum in two steps. The first step is to construct the pull-back diagram

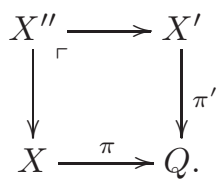

The DG module $X^{\prime \prime}$ is a submodule of the direct $\operatorname{sum} X \oplus X^{\prime}$, so each $X_{i}^{\prime \prime}$ is the submodule of

$$
\left(X \oplus X^{\prime}\right)_{i}=X_{i} \oplus X_{i}^{\prime} \cong N_{i} \oplus Q_{i} \oplus N_{i} \oplus Q_{i}
$$

consisting of all vectors $\left[\begin{array}{c}x \\ x^{\prime}\end{array}\right]$ such that $\pi_{i}^{\prime}\left(x^{\prime}\right)=\pi_{i}(x)$, that is, all vectors of the form $\left[\begin{array}{llll}p & q & p^{\prime} & q^{\prime}\end{array}\right]^{T}$ such that $q=q^{\prime}$. In other words, we have

$$
N_{i} \oplus Q_{i} \oplus N_{i} \stackrel{\cong}{\longrightarrow} X_{i}^{\prime \prime}
$$

where the isomorphism is given by $\left[\begin{array}{lll}p & q & p^{\prime}\end{array}\right]^{T} \mapsto\left[\begin{array}{llll}p & q & p^{\prime} & q\end{array}\right]^{T}$. The differential on $X \oplus X^{\prime}$ is the natural diagonal map. So, under the isomorphism (3.5.1), the differential on $X^{\prime \prime}$ has the form

$$
X_{i}^{\prime \prime} \cong N_{i} \oplus Q_{i} \oplus N_{i} \stackrel{\partial_{i}^{X^{\prime \prime}}=\left[\begin{array}{ccc}
\partial_{i}^{N} & \lambda_{i} & 0 \\
0 & \partial_{i}^{Q} & 0 \\
0 & \lambda_{i}^{\prime} & \partial_{i}^{N}
\end{array}\right]}{\longrightarrow} N_{i-1} \oplus Q_{i-1} \oplus N_{i-1} \cong X_{i-1}^{\prime \prime} .
$$

The next step in the construction of $\zeta+\zeta^{\prime}$ is to build $\tilde{X}$, which is the cokernel of the morphism $\gamma: N \rightarrow X^{\prime \prime}$ given by $p \mapsto\left[\begin{array}{c}-p \\ 0 \\ p\end{array}\right]$. That is, since $\gamma$ is injective, the complex $\widetilde{X}$ is determined by the exact sequence $0 \rightarrow N \stackrel{\gamma}{\rightarrow} X^{\prime \prime} \stackrel{\tau}{\rightarrow} \widetilde{X} \rightarrow 0$. It is straightforward to show that this sequence has the following form

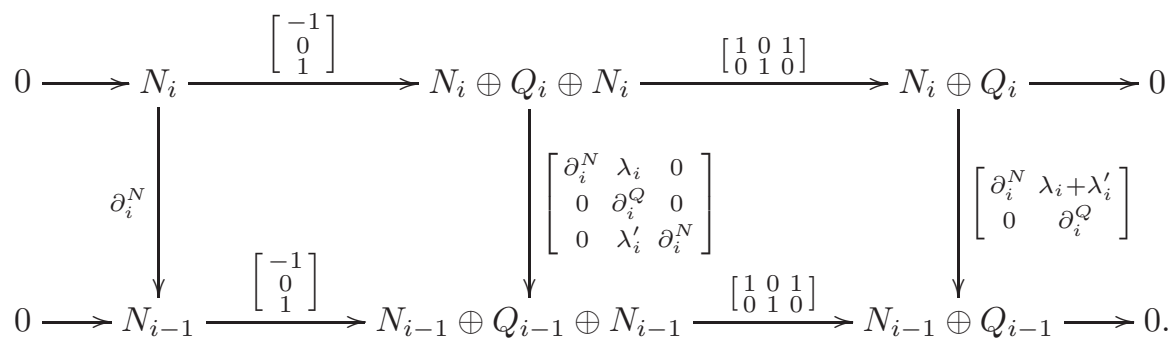

By inspecting the right-most column of this diagram, we see that $\widetilde{X}$ has the desired form. Furthermore, checking the module structures at each step of the construction, we see that the scalar multiplication on $\tilde{X}$ is the natural one $a\left[\begin{array}{c}p \\ q\end{array}\right]=\left[\begin{array}{l}a p \\ a q\end{array}\right]$. This concludes the proof of Claim 1 . 
Claim 2. $\Psi$ is injective. Suppose that $\zeta \in \operatorname{Ker}(\Psi)$ is represented by the displays 3.3.1 - 3.3.3). The condition $\Psi(\zeta)=0$ says that $\lambda \in \operatorname{Im}\left(\partial_{0}^{\operatorname{Hom}_{A}(Q, N)}\right)$, so there is an element $s \in \operatorname{Hom}_{A}(Q, N)_{0}$ such that $\lambda=\partial_{0}^{\operatorname{Hom}_{A}(Q, N)}(s)$. Thus, for each $i$ we have $\lambda_{i}=\partial_{i}^{N} s_{i}-s_{i-1} \partial_{i}^{Q}$. From this, it is straightforward to show that the following diagram commutes:

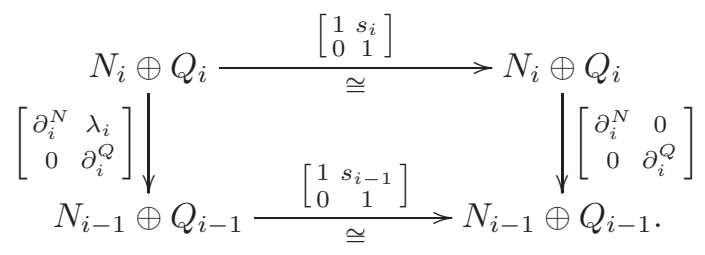

From the fact that $s$ is $A$-linear, it follows that the maps $\left[\begin{array}{cc}1 & s_{i} \\ 0 & 1\end{array}\right]$ describe an $A$-linear isomorphism $X \cong N \oplus Q$ making the following diagram commute:

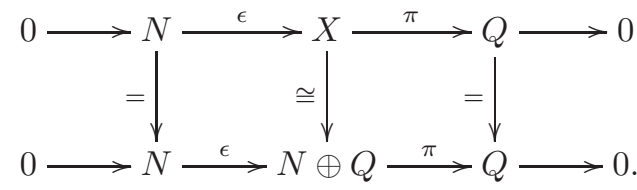

In other words, the sequence (3.3.1) splits, so we have $\zeta=0$, and $\Psi$ is injective. This concludes the proof of Claim 2

Claim 3. $\Psi$ is surjective. For this, let $\xi \in \mathrm{H}_{-1}\left(\operatorname{Hom}_{A}(Q, N)\right)$ be represented by $\lambda \in \operatorname{Ker}\left(\partial_{-1}^{\operatorname{Hom}_{A}(Q, N)}\right)$. Using the fact that $\lambda$ is $A$-linear such that $\partial_{-1}^{\operatorname{Hom}_{A}(Q, N)}(\lambda)=$ 0 , one checks directly that the displays 3.3.2-3.3.3 describe an exact sequence of DG $A$-module homomorphisms of the form 3.3.1 whose image under $\Psi$ is $\xi$. This concludes the proof of Claim 3 and the proof of the theorem.

Remark 3.6. After the results of this paper were announced, Avramov, et al. [2] established the following generalization of Theorem 3.5

Proposition 3.7. Let $A$ be a $D G R$-algebra, and let $M$ and $N$ be $D G A$-modules. There is a monomorphism of abelian groups

$$
\kappa: \mathrm{H}_{0}\left(\operatorname{Hom}_{A}\left(\Sigma^{-1} M, N\right)\right) \rightarrow \operatorname{YExt}_{U}^{1}(M, N)
$$

with image equal to the set of equivalence classes of graded-split exact sequences of the form $0 \rightarrow N \rightarrow X \rightarrow M \rightarrow 0$.

To see how this generalizes Theorem 3.5, first note that if $M$ is graded-projective, then the map $\kappa$ is bijective, as in this case every element of $\operatorname{YExt}_{U}^{1}(M, N)$ is gradedsplit; thus, we have $\mathrm{H}_{-1}\left(\operatorname{Hom}_{A}(M, N)\right) \cong \mathrm{H}_{0}\left(\operatorname{Hom}_{A}\left(\Sigma^{-1} M, N\right)\right) \cong \operatorname{YExt}_{U}^{1}(M, N)$.

Proof 3.8 (Proof of Theorem $\mathrm{A}$ ). Using Theorem 3.5 we need only justify the isomorphism $\operatorname{YExt}_{A}^{i}(Q, N) \cong \operatorname{Ext}_{A}^{i}(Q, N)$ for $i \geqslant 2$. Let

$$
L_{\bullet}^{+}=\cdots \stackrel{\partial_{2}^{L}}{\longrightarrow} L_{1} \stackrel{\partial_{1}^{L}}{\longrightarrow} L_{0} \stackrel{\pi}{\rightarrow} Q \rightarrow 0
$$

be a resolution of $Q$ by categorically projective DG $A$-modules. Since each $L_{j}$ is categorically projective, we have $\operatorname{YExt}_{A}^{i}\left(L_{j},-\right)=0$ for all $i \geqslant 1$ and $L_{j} \simeq 0$ for each $j$, so we have $\operatorname{Ext}_{A}^{i}\left(L_{j},-\right)=0$ for all $i$. Set $Q_{i}:=\operatorname{Im} \partial_{i}^{L}$ for each $i \geqslant 1$. Each 
$L_{i}$ is graded-projective, so the fact that $Q$ is graded-projective implies that each $Q_{i}$ is graded-projective.

Now, a straightforward dimension-shifting argument explains the first and third isomorphisms in the following display for $i \geqslant 2$ :

$$
\operatorname{YExt}_{A}^{i}(Q, N) \cong \operatorname{YExt}_{A}^{1}\left(Q_{i-1}, N\right) \cong \operatorname{Ext}_{A}^{1}\left(Q_{i-1}, N\right) \cong \operatorname{Ext}_{A}^{i}(Q, N) .
$$

The second isomorphism is from Theorem 3.5 since each $Q_{i}$ is graded-projective.

The next example shows that one can have $\operatorname{YExt}_{A}^{0}(Q, N) \nRightarrow \operatorname{Ext}_{A}^{0}(Q, N)$, even when $Q$ is semi-free.

Example 3.9. Continue with the assumptions and notation of Example 3.1, and set $Q=N=\underline{R}$. It is straightforward to show that the morphisms $\underline{R} \rightarrow \underline{R}$ are precisely given by multiplication by fixed elements of $R$, so we have the first step in the next display:

$$
\operatorname{YExt}_{A}^{0}(\underline{R}, \underline{R}) \cong R \neq 0=\operatorname{Ext}_{A}^{0}(\underline{R}, \underline{R}) .
$$

The third step follows from the condition $\underline{R} \simeq 0$.

Remark 3.10. It is perhaps worth noting that our proofs can also be used to give the isomorphisms from Theorem $\mathrm{A}$ when $Q$ is not necessarily semi-projective, but $N$ is "semi-injective".

\section{YExt $^{1}$ AND TRUNCATIONS}

For our work in [12, we need to know how YExt respects the following notion.

Definition 4.1. Let $A$ be a DG $R$-algebra, and let $M$ be a DG $A$-module. Given an integer $n$, the $n$th soft left truncation of $M$ is the complex

$$
\tau(M)_{(\leqslant n)}:=\cdots \rightarrow 0 \rightarrow M_{n} / \operatorname{Im}\left(\partial_{n+1}^{M}\right) \rightarrow M_{n-1} \rightarrow M_{n-2} \rightarrow \cdots
$$

with differential induced by $\partial^{M}$. In other words, $\tau(M)_{(\leqslant n)}$ is the quotient DG $A$-module $M / M^{\prime}$ where $M^{\prime}$ is the following DG submodule of $M$ :

$$
M^{\prime}=\cdots \rightarrow M_{n+2} \rightarrow M_{n+1} \rightarrow \operatorname{Im}\left(\partial_{n+1}^{M}\right) \rightarrow 0 .
$$

Note that we have $M^{\prime} \simeq 0$ if and only if $n \geqslant \sup (M)$, so the natural morphism $\rho: M \rightarrow \tau(M)_{(\leqslant n)}$ of DG $A$-modules yields an isomorphism in $\mathcal{D}(A)$ if and only if $n \geqslant \sup (M)$.

Proposition 4.2. Let $A$ be a $D G R$-algebra, and let $M$ and $N$ be $D G A$-modules. Assume that $n$ is an integer such that $N_{i}=0$ for all $i>n$. Then the natural map $\operatorname{YExt}_{A}^{1}\left(\tau(M)_{(\leqslant n)}, N\right) \rightarrow \operatorname{YExt}_{A}^{1}(M, N)$ induced by the morphism $\rho: M \rightarrow$ $\tau(M)_{(\leqslant n)}$ from Definition 4.1 is a monomorphism.

Proof. Let $\Upsilon$ denote the map $\operatorname{YExt}_{A}^{1}\left(\tau(M)_{(\leqslant n)}, N\right) \rightarrow \operatorname{YExt}_{A}^{1}(M, N)$ induced by $\rho$. Let $\alpha \in \operatorname{Ker}(\Upsilon) \subseteq \operatorname{YExt}_{A}^{1}\left(\tau(M)_{(\leqslant n)}, N\right)$ be represented by the exact sequence

$$
0 \rightarrow N \stackrel{f}{\rightarrow} X \stackrel{g}{\rightarrow} \tau(M)_{(\leqslant n)} \rightarrow 0 .
$$

Note that, since $N_{i}=0=\left(\tau(M)_{(\leqslant n)}\right)_{i}$ for all $i>n$, we have $X_{i}=0$ for all $i>n$. Our assumptions imply that $0=\Upsilon([\alpha])=[\beta]$ where $\beta$ comes from the following 
pull-back diagram:

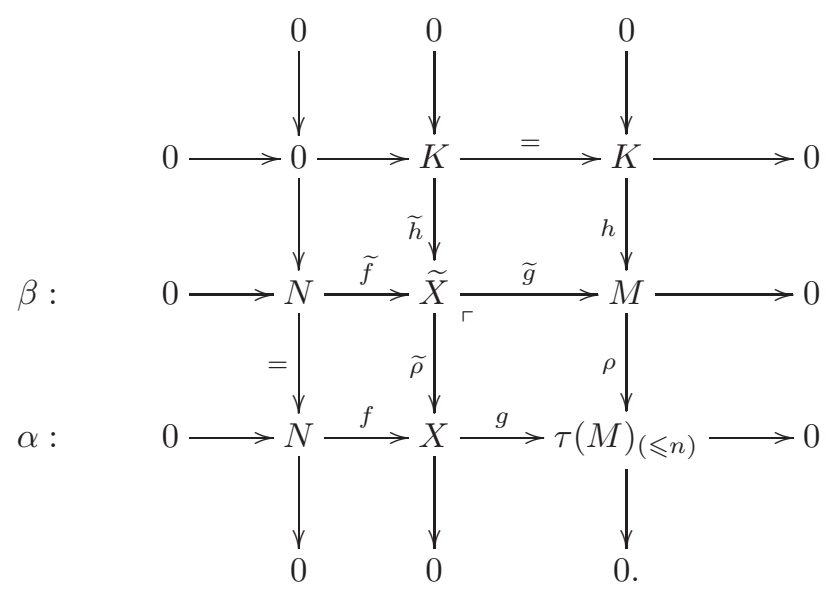

The middle row $\beta$ of this diagram is split exact since $[\beta]=0$, so there is a morphism $F: \widetilde{X} \rightarrow N$ of DG $A$-modules such that $F \circ \widetilde{f}=\operatorname{id}_{N}$. Note that $K$ has the form

$$
K=\cdots \stackrel{\partial_{n+2}^{M}}{\longrightarrow} M_{n+1} \stackrel{\partial_{n+1}^{M}}{\longrightarrow} \operatorname{Im}\left(\partial_{n+1}^{M}\right) \rightarrow 0
$$

because of the right-most column of the diagram.

We claim that $F \circ \widetilde{h}=0$. It suffices to check this degree-wise. When $i>n$, we have $N_{i}=0$, so $F_{i}=0$, and $F_{i} \circ \widetilde{h}_{i}=0$. When $i<n$, the display (4.2.3) shows that $K_{i}=0$, so $\widetilde{h}_{i}=0$, and $F_{i} \circ \widetilde{h}_{i}=0$. For $i=n$, we first note that the display (4.2.3) shows that $\partial_{n+1}^{K}$ is surjective. In the following diagram, the faces with solid arrows commute because $\widetilde{h}$ and $F$ are morphisms:

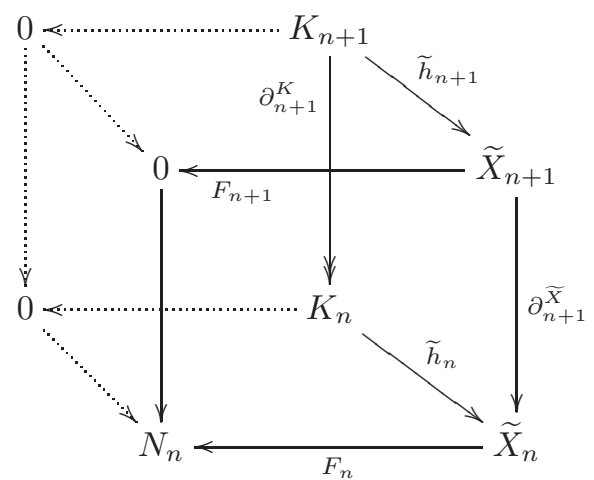

Since $\partial_{n+1}^{K}$ is surjective, a simple diagram chase shows that $F_{n} \circ \widetilde{h}_{n}=0$. This establishes the claim.

To conclude the proof, note that the previous claim shows that the map $K \rightarrow 0$ is a left-splitting of the top row of diagram (4.2.2) that is compatible with the left-splitting $F$ of the middle row. It is now straightforward to show that $F$ induces a morphism $\bar{F}: X \rightarrow N$ of DG $A$-modules that left-splits the bottom row of diagram (4.2.2). Since this row represents $\alpha \in \operatorname{YExt}_{A}^{1}\left(\tau(M)_{(\leqslant n)}, N\right)$, we conclude that $[\alpha]=0$, so $\Upsilon$ is a monomorphism. 
The next example shows that the monomorphism from Proposition 4.2 may not be an isomorphism.

Example 4.3. Continue with the assumptions and notation of Example 3.1 The following diagram describes a non-zero element of $\operatorname{YExt}_{R}^{1}(M, N)$ :
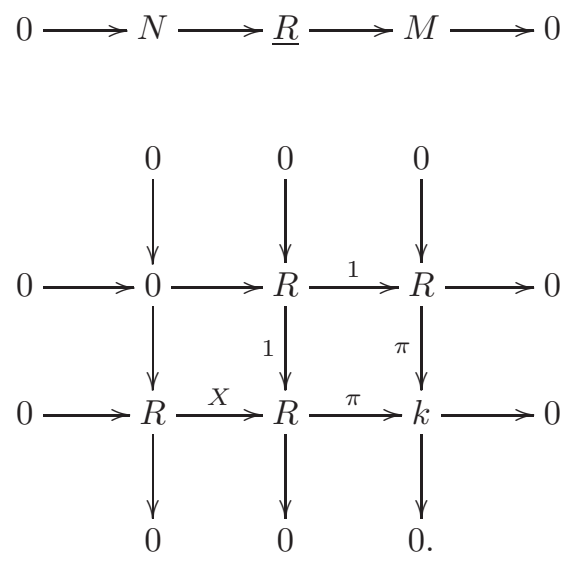

It is straightforward to show that $\tau(M)_{(\leqslant 0)}=0$, so we have

$$
0=\mathrm{YExt}_{A}^{1}\left(\tau(M)_{(\leqslant 0)}, N\right) \hookrightarrow \operatorname{YExt}_{A}^{1}(M, N) \neq 0
$$

thus this map is not an isomorphism.

Proposition 4.4. Let $A$ be a $D G R$-algebra, and let $C$ be a semi-projective $D G$ $A$-module such that $\operatorname{Ext}_{R}^{1}(C, C)=0$. For $n \geqslant \sup (C)$, one has

$$
\mathrm{YExt}_{A}^{1}(C, C)=0=\mathrm{YExt}_{A}^{1}\left(\tau(C)_{(\leqslant n)}, \tau(C)_{(\leqslant n)}\right) .
$$

Proof. From Theorem 3.5, we have $\operatorname{YExt}_{A}^{1}(C, C) \cong \operatorname{Ext}_{A}^{1}(C, C)=0$. For the remainder of the proof, assume without loss of generality that $\sup (C)<\infty$. Another application of Theorem 3.5 explains the first step in the next display:

$$
\mathrm{YExt}_{A}^{1}\left(C, \tau(C)_{(\leqslant n)}\right) \cong \operatorname{Ext}_{A}^{1}\left(C, \tau(C)_{(\leqslant n)}\right) \cong \operatorname{Ext}_{A}^{1}(C, C)=0 .
$$

The second step comes from the assumption $n \geqslant \sup (C)$ which guarantees that the natural morphism $C \rightarrow \tau(C)_{(\leqslant n)}$ represents an isomorphism in $\mathcal{D}(A)$. Proposition 4.2 implies that $\operatorname{YExt}_{A}^{1}\left(\tau(C)_{(\leqslant n)}, \tau(C)_{(\leqslant n)}\right)$ is isomorphic to a subgroup of $\mathrm{YExt}_{A}^{1}\left(C, \tau(C)_{(\leqslant n)}\right)=0$, so $\operatorname{YExt}_{A}^{1}\left(\tau(C)_{(\leqslant n)}, \tau(C)_{(\leqslant n)}\right)=0$, as desired.

\section{ACKNowledgments}

We are grateful to Luchezar Avramov for helpful discussions about this work.

\section{REFERENCES}

1. L. L. Avramov, Infinite free resolutions, Six lectures on commutative algebra (Bellaterra, 1996), Progr. Math., vol. 166, Birkhäuser, Basel, 1998, pp. 1-118. MR 99m:13022

2. L. L. Avramov, H.-B. Foxby, and S. Halperin, Differential graded homological algebra, in preparation.

3. L. L. Avramov, H.-B.Foxby, and J. Lescot, Bass series of local ring homomorphisms of finite flat dimension, Trans. Amer. Math. Soc. 335 (1993), no. 2, 497-523. MR 93d:13026

4. R. Baer, Erweiterung von Gruppen und ihren Isomorphismen, Math. Z. 38 (1934), no. 1, 375-416. MR 1545456 
5. K. Beck and S. Sather-Wagstaff, A somewhat gentle introduction to differential graded commutative algebra, Connections Between Algebra, Combinatorics, and Geometry, Proceedings in Mathematics and Statistics, vol. 76, Springer, New York, Heidelberg, Dordrecht, London, 2014, pp. 3-99.

6. L. W. Christensen, Gorenstein dimensions, Lecture Notes in Mathematics, vol. 1747, SpringerVerlag, Berlin, 2000. MR 2002e:13032

7. L. W. Christensen, H.-B. Foxby, and H. Holm, Derived category methods in commutative algebra, in preparation.

8. Y. Félix, S. Halperin, and J.-C. Thomas, Rational homotopy theory, Graduate Texts in Mathematics, vol. 205, Springer-Verlag, New York, 2001. MR 1802847

9. S. I. Gelfand and Y. I. Manin, Methods of homological algebra, Springer-Verlag, Berlin, 1996. MR 2003m:18001

10. R. Hartshorne, Residues and duality, Lecture Notes in Mathematics, No. 20, Springer-Verlag, Berlin, 1966. MR 36 \#5145

11. B. Keller, Deriving DG categories, Ann. Sci. École Norm. Sup. (4) 27 (1994), no. 1, 63-102. MR 1258406 (95e:18010)

12. S. Nasseh and S. Sather-Wagstaff, Geometric aspects of representation theory for DG algebras: answering a question of Vasconcelos, preprint (2012), arXiv:1201.0037.

13. J.-L. Verdier, Catégories dérivées, SGA $4 \frac{1}{2}$, Springer-Verlag, Berlin, 1977, Lecture Notes in Mathematics, Vol. 569, pp. 262-311. MR 57 \#3132

14. - Des catégories dérivées des catégories abéliennes, Astérisque (1996), no. 239, xii+253 pp. (1997), With a preface by Luc Illusie, Edited and with a note by Georges Maltsiniotis. MR 98c: 18007

15. C. A. Weibel, An introduction to homological algebra, Cambridge Studies in Advanced Mathematics, vol. 38, Cambridge University Press, Cambridge, 1994. MR 1269324 (95f:18001)

Saeed Nasseh, Department of Mathematical Sciences, Georgia Southern University, Statesboro, Georgia 30460, USA

E-mail address: snasseh@georgiasouthern.edu

$U R L:$ https://cosm.georgiasouthern.edu/math/saeed.nasseh

Sean Sather-Wagstaff, Department of Mathematical Sciences, Clemson University, O-110 Martin Hall, Box 340975, Clemson, S.C. 29634, USA

E-mail address: ssather@clemson.edu

URL: https://ssather.people.clemson.edu/ 